\title{
BROWN- AND RED-PIGMENTED PSEUDOMONAS AERUGINOSA: DIFFERENTIATION BETWEEN MELANIN AND PYORUBRIN
}

\author{
J. Ogunnariwo and J. M. T. Hamilton-Miller \\ Department of Medical Microbiology, Royal Free Hospital, \\ Gray's Inn Road, London, WCI
}

Plate XII

WE have recently isolated from clinical material three strains of Pseudomonas aeruginosa that produced a brown pigment which diffused through agar. Pigmentation varied in intensity from light brown to a deep coffee colour. Perusal of several standard microbiology textbooks and a survey of the literature left us uncertain whether this pigment was one of those occasionally produced by $\boldsymbol{P}$. aeruginosa, namely pyorubrin and pyomelanin. Consequently, we sought to obtain for comparative purposes strains of this species authenticated as producers of one or other of these pigments. It soon became apparent, however, that some of the characteristics attributed to certain standard strains from the National Collection of Type Cultures (NCTC) were in this respect misleading, and it was not until we had obtained further reference strains from Japan that we were able to define our pigment categorically.

The appearance in culture of $\boldsymbol{P}$. aeruginosa strains that produce red pigment have been described by some observers simply as "red" (Meader, Robinson and Leonard, 1925; Wahba, 1965) and by others as yellowish turning gradually to red (Gessard, 1920; Holliman, 1961; Narano, 1965). The appearance is certainly strongly dependent upon cultural conditions and the production of other pigments (Simon, 1956). The position is complicated by the fact that acidification of pyocyanin produces a red colour, and that exposure of plates containing pyocyanin to light and air for some time ( 8 days, in our hands) produces a light brown colour. Some authorities, indeed, appear to regard all red and brown pigments of $P$. aeruginosa as oxidation products of pyocyanin (Leonard, 1924; Wilson and Miles, 1964). The frequency of red strains among clinically isolated specimens is low; Wahba (1965) reported 3.5\% -more than half obtained from infected urines; and Phillips (1969) found 6\%.

The cultural appearance of strains of $P$. aeruginosa that produce brown pigment is also dependent upon the nature of the medium. It has been described variously as "coffeebrown" (Yabuuchi and Ohyama, 1972), "brown, dark brown or black" (Elston, 1968) and "rouge-brun" (Gessard, 1920). Some strains have also been referred to merely as being "melanogenic" (Gessard, 1925; Espinosa, Portoles and Hidalgo, 1970). The frequency of so-called brown strains among clinically isolated $P$. aeruginosa has been variously assessed as $2.5 \%$ (Elston, 1968), 10.5\% (Wahba, 1965) and $<1 \%$ (Phillips, 1969).

It is clear from the above that there may be considerable difficulty in deciding whether a light mahogany shade, such as that produced by our strains on Sensitivity-Test Agar (especially after a short period of incubation) should best be described as " reddish brown" (i.e., pyorubrin) or "light brown" (i.e., pyomelanin). We have also observed that several experienced microbiologists were erroneously under the impression that melanin is black, as opposed to dark brown. This is hardly surprising in view of the derivation of the word (Greek, $\mu \varepsilon \lambda \alpha \varsigma=$ black); further, melanin is described in The Shorter Oxford English Dictionary (1965) as being black. So it seems likely that intermediate shades of brown may not always be accepted as being due to melanin; further, there is definite evidence that pyomelanin has been in the past misidentified as pyorubrin (Yabuuchi and Ohyama, 1972).

Our purpose in this paper, then, is to describe a simple cultural method for the differentia-

Received 4 June 1974; accepted 4 Aug. 1974.

J. MED. MICROBIOL.-VOL. 8 (1975) 
tion of pyorubrin and pyomelanin, to give a brief description of our pyomelanin-producing strains, and to correct certain misleading descriptions in the 1972 Catalogue of the NCTC.

\section{MATERIALS AND METHODS}

Bacterial strains. P. aeruginosa strains 355, 902 and 908 were isolated in these laboratories, respectively from a urine sample, a specimen of bile and a wound swab; under appropriate conditions (see Results section), each produced a diffusible, mahoganycoloured pigment. The reference strain for pyomelanin production was no. NCTC6749, shown to produce this pigment by Yabuuchi and Ohyama (1972), despite being listed in the NCTC catalogue as "var. erythrogenes". This varietal epithet, whose origin is not known, suggests the production of a red pigment. In view of this, we also obtained other strains listed as $P$. aeruginosa var. erythrogenes from the NCTC, nos. 10561 and 5083, together with the alleged pyorubrin-producing strain NCTC10299. Dr Eiko Yabuuchi very kindly supplied three pyorubrin-producing strains, nos. KM145 (Narano, 1965), KM352 and KM360 (Yabuuchi and Ohyama, 1972). Aeromonas salmonicida, no. NCTC10402, which is identified by virtue of its production of a brown pigment (Griffin, Snieszko and Friddle, 1953), was also studied. We classified our clinically isolated strains of $P$. aeruginosa as recommended by Phillips (1969): phage and serological typing were performed at the Cross-Infection Reference Laboratory, Colindale (we are very grateful to Dr M. T. Parker for this service).

Media and cultural examinations. Colour typing (Gessard, 1920) was performed, basically as described by Simon (1956), on the following media: (1) Lab-Lemco (Oxoid) $1 \%(\mathrm{w} / \mathrm{v})$, (2) Bacto-Tryptone (Difco) $2 \%(w / v)$, and (3) Bacto-Tryptone $2 \%(w / v)$ and glycerol $2.5 \%$ ( $\mathrm{v} / \mathrm{v})$, each with the addition of agar $1.5 \%(\mathrm{w} / \mathrm{v})$. Minimal salts medium (MSM) was as described by Davis and Mingioli (1950); it was supplemented where indicated with L-tyrosine or DL-glutamic acid 1\%(w/v). Furunculosis Agar was purchased from Difco; it has the following composition: Tryptone $10 \mathrm{~g}$, yeast extract $5 \mathrm{~g}$, L-tyrosine $1 \mathrm{~g}, \mathrm{NaCl} 2.5 \mathrm{~g}$, agar $15 \mathrm{~g}$ per litre. A. salmonicida was grown at $22^{\circ} \mathrm{C}$, other organisms at $37^{\circ} \mathrm{C}$. For chemical and physical studies, pyomelanin was produced as follows: 20-ml amounts of Hartley digest broth (Southern Group Laboratories, London, SE13) were inoculated and incubated at $37^{\circ} \mathrm{C}$ for $48 \mathrm{~h}$. Each culture was then poured into a petri dish $(8 \cdot 5-\mathrm{cm}$ diameter) and incubated for a further 3 days at room temperature on the bench with occasional swirling. Organisms were removed by centrifugation and the supernatant fraction, crude pyomelanin, was stored at $4^{\circ} \mathrm{C}$. Animal melanin was obtained from Koch-Light Laboratories Ltd, Colnbrook, Bucks SL3 OBZ.

\section{RESULTS}

Production of pigment under routine conditions. The three strains isolated in this laboratory were first recognised as producing a brownish-red pigment during routine sensitivity testing on Oxoid Sensitest Agar. Plates examined directly after removal from the incubator showed very slight pigmentation if any; after about $30 \mathrm{~min}$. on the bench, however, the medium directly around the colonies had started to change by way of a pinkish-brown to a dark mahogany colour, which rapidly diffused throughout the medium within a few hours. Colourless plates transferred directly from the incubator to a transparent anaerobic jar (Gaspak system, BBL) exposed to the light did not show pigmentation; thus it is clear that the final stages of pigment production are dependent upon both light and oxygen. Pigment production was not significant on MacConkey agar or blood agar, nor in liquid culture. It was noticeable that pigment production was enhanced after five or six subcultures in the laboratory. Of non-routine media used, Oxoid Nutrient Agar no. 2 was more conducive to pigment production than was Sensitest Agar, and Furunculosis Agar was still more so.

Identification. Our three strains (nos. 355, 902 and 908) were readily identifiable as $P$. aeruginosa. Unlike the "melanogenic" strains of Espinosa et al. (1970) they were not haemolytic, and unlike the brown strains of Inoue (1961) and Liu (1962) they were motile. Strain 355 was of serotype 6 , while strains 902 and 908 were auto-agglutinable; all three 
were untypable by phage. No. NCTC6749 was of serotype 6, and had the phage-typing pattern 7/44/109/M4. Strains 355 and 902 were of Gessard colour-type MS, and strain 908 was untypable by this means.

Comparison with known pyorubrin- and pyomelanin-producing strains. In all respects our three brown strains behaved in the same way as did the standard pyomelanin-producing strain NCTC6749. Therefore we conclude that the pigment elaborated by our three strains is pyomelanin. The two other strains labelled by the National Collection as "var. erythrogenes"-nos. NCTC 5083 and 10561-were also found to produce pyomelanin.

The three reference pyorubrin-producing strains, nos. 145, 352 and 360, all behaved in a consistent way, which differed from that of the pyomelanin-producing strains. The strain described in the NCTC catalogue as producing pyorubrin, no. NCTC10299, did not conform to the pattern shown by the other pyorubrin-producing strains, and in fact produced green pigment only. It was of Gessard colour type PeS.

Differentiation between pyorubrin and pyomelanin could be made in the following way. All the strains that formed brown or reddish pigments on complex solid media grew but remained colourless on MSM; supplementing this medium with $1 \% \mathrm{~L}$-tyrosine enhanced the production of melanin but not of pyorubrin. Addition of $1 \% \mathrm{DL}$-glutamate to MSM enabled the pyorubrin-producing strains to produce their pigment (Narano, 1966) while the melanogenic strains remained colourless. In liquid MSM (10 ml-volumes in 30-ml screw-capped "Universal" containers), pigment production was extremely poor despite appropriate supplementation. On Furunculosis Agar, melanin production was very marked, while pyorubrin production was poor (the figure). A. salmonicida, no. NCTC10402, which grew extremely poorly and failed to pigment at $37^{\circ} \mathrm{C}$, produced brown pigment after about $48 \mathrm{~h}$ at room temperature on Furunculosis Agar, while the melanogenic $P$. aeruginosa strains had started to go brown under these conditions after about $18 \mathrm{~h}$.

Properties of pyomelanin. Pyomelanin showed an absorption spectrum qualitatively identical with that of melanin from an animal source (Schmidli, 1955). It was not extracted from solution by ether, chloroform and amyl alcohol, and was precipitated by acidification. In the series of tests described by Bull (1970) for the characterisation of melanins, pyomelanin and the animal melanin behaved in similar ways. Pyomelanin did not conform to the chemical properties of crude pyorubrin as described by Meader et al. (1925).

Antibiotic sensitivities of melanogenic strains. Five melanin-producing strains of $P$. aeruginosa were tested by the disk method against gentamicin $(10 \mu \mathrm{g})$, cephalothin $(15 \mu \mathrm{g})$, carbenicillin $(100 \mu \mathrm{g})$, co-trimoxazole $(25 \mu \mathrm{g})$, ampicillin $(10 \mu \mathrm{g})$, tetracycline $(10 \mu \mathrm{g})$, streptomycin $(25 \mu \mathrm{g})$, cephalexin $(30 \mu \mathrm{g})$, chloramphenicol $(25 \mu \mathrm{g})$ and polymyxin B $(100 \mu \mathrm{g})$, on Sensitest Agar. All were sensitive to gentamicin and polymyxin B, and four to carbenicillin; in addition, four were sensitive to streptomycin, two to tetracyline, two to co-trimoxazole and one to ampicillin. Sensitivity to the last four agents is unusual among our routine isolates of $P$. aeruginosa, so it appears that melanin-producing strains may be more senstitive to antibiotics than are other strains (see Yabuuchi and Ohyama, 1972).

\section{Discussion}

The $P$. aeruginosa strains NCTC5083, 6749 and 10561, all labelled as " var. erythrogenes" in the NCTC catalogue, in fact produce pyomelanin and not pyorubrin. We suggest that this dubious epithet, the origin of which is not known (L. R. Hill, personal communication), be abandoned. It does not seem from our results and from those of others (Yabuuchi and Ohyama, 1972) that pyomelanin-producing strains are sufficiently different to warrant a varietal epithet such as " melanogenes". Strain NCTC10299, described as producing pyorubrin, does not in fact do so.

We have shown that pyomelanin production is enhanced on Furunculosis Agar; pyorubrin production, however, is only slight under these conditions. Thus, this medium should be useful in the identification of the pigment produced by strains of $P$. aeruginosa that appear reddish brown on certain routine media (typically, Sensitivity-Test Agar).

Furunculosis Agar, which contains $0 \cdot 1 \%$ L-tyrosine, was originally designed to enhance 
pigment production in $A$. salmonicida. This organism is oxidase positive, and there appears to be some confusion between the purple colour resulting from a positive oxidase test and the brown colour of the pigment (Griffin et al., 1953; Difco Supplementary Literature, 1968). We chose this medium, probably unfamiliar to most medical microbiologists, because of the reported enhancing effect of tyrosine on pyomelanin production (Yabuuchi and Ohyama, 1972). Our results suggest that caution must be exercised in the presumption (Difco Supplementary Literature, 1968) that brown-pigmenting strains on this medium are $A$. salmonicida; motility testing, as recommended by Griffin et al. (1953), is a necessary second step.

The occurrence of brown (pyomelanin-producing) strains of $P$. aeruginosa was originally reported in France in 1897 (see Gessard, 1901), and is now well documented in several other countries-Germany (Mann, 1969), USA (Elston, 1968) and Japan (Yabuuchi and Ohyama, 1972), for example. The major study in this country has been that by Wahba (1965), but it is not entirely clear whether pyorubrin and pyomelanin could be differentiated in every case by the techniques he used, so the percentage of brown strains quoted by him should be regarded with some caution.

Pyomelanin seems chemically unrelated to animal melanin (DOPA or indole melanin) although their absorption spectra in our hands appear to be identical. The experiments of Yabuuchi and Ohyama (1972) and of Mann (1969) suggest that pyomelanin may be a catechol melanin; this would fit with the suggestion of Nicolaus, Piattelli and Fattorusso (1964) that, while melanins from animals are based on indole, those from the vegetable kingdom appear to be catechol melanins.

Erythrogenic strains of $P$. aeruginosa were first described in 1919 (see Gessard, 1920) and the pigment-pyorubrin-was first examined in detail by Meader et al. (1925). Several authors have referred to this pigment, incorrectly, as "pyorubin". A comparison of the absorption spectrum of pyorubrin (Narano, 1966) with that of aeruginosins A and B (Holliman, 1961) allows us to conclude that pyorubrin is a mixture of the latter pigments. Pyorubrin is, therefore, like pyocyanin, a phenazine pigment.

\section{SUMMARY}

The pigment produced by three clinically isolated strains of Pseudomonas aeruginosa has been shown to be pyomelanin. This pigment is also produced by three strains of the same species labelled as "var. erythrogenes" by the National Collection of Type Cultures, and by Aeromonas salmonicida. Melanin and pyorubrin may be distinguished by the differential effects of tyrosine and glutamate on their production in minimal salts medium; Furunculosis Agar is a suitable medium for differentiation of these pigments.

We are grateful to the Wellcome Trust for a grant enabling the publication of the colour plate.

\section{REFERENCES}

Bull, A. T. 1970. Chemical composition of wild-type and mutant Aspergillus nidulans cell walls. The nature of polysaccharide and melanin constituents. J.gen. Microbiol., 63, 75 .

Davis, B. E. AND Mingioli, E. S. 1950. Mutants of Escherichia coli requiring methionine or vitamin B12. J. Bact., 60, 17.

DIFCO SUPPLEMENTARY LITERATURE. 1968. Furunculosis agar, Michigan, p. 159.

ELsTon, H. R. 1968. Melanogenic strains of Pseudomonas aeruginosa in biological specimens. Amer. J. med. Technol., 34, 189.

Espinosa, M., Portoles, A. ANd Hidalgo, A. 1970. Chromogenesis variations in pathogenic Pseudomonas aeruginosa strains evaluated by the culture media. Zentbl Bakt. (Naturwiss.), 125, 597.

GessarD, C. 1901. Variété mélanogène du bacille pyocyanique. Annls Inst. Pasteur, Paris, 15, 817. 
BROWN- AND RED-PIGMENTED $P$. AERUGINOSA
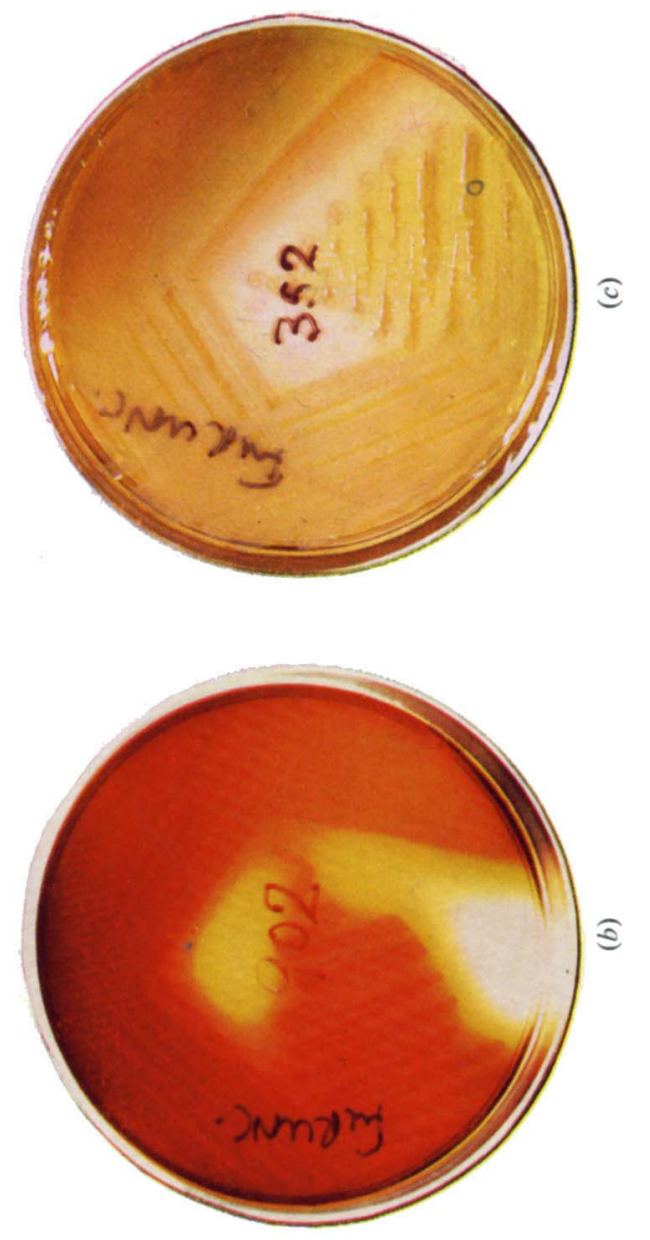

nin

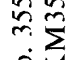

응

ธิ

茎

$\Xi \ddot{E}$

(气

응

¿ $\approx$

ธั

5

$\Xi$

范

등

突

$<0$

$\because \overrightarrow{0}$

응

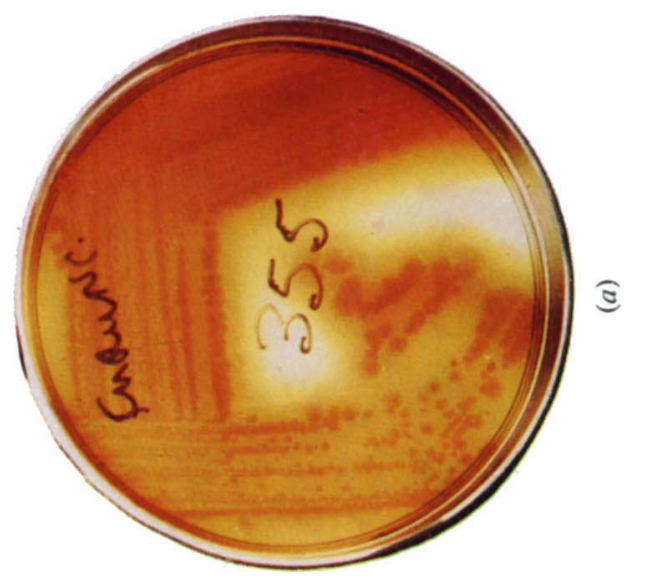

志艺

ㄷํㅇ

응

过

สํำ

¿

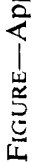


Gessard, C. 1920. Technique d'identification des germes pyocyaniques. Annls Inst. Pasteur, Paris, 34, 88.

Gessard, C. 1925. Bacilles pyocyanoides de la variété mélanogène. $C . r$. hebd. Séanc. Acad. Sci., Paris, 180, 972.

Griffin, P. J., Snieszko, S. F. ANd Friddle, S. B. 1953. A new adjuvant in the diagnosis of fish furunculosis caused by Bacterium salmonicida. Vet. Med., 48, 280.

Hollman, F. G. 1961. Some bacterial pigments (phenazines). S. Afr. ind. Chem., 15, 233.

INOUE, E. 1961. Studies on mutations of Pseudomonas aeruginosa. J. Am. med. Womens' Ass., 16, 858.

Leonard, V. 1924. Pyorubrin, a new pigment produced by Bacillus pyocyaneus. Amer. J. Hyg., 4, 404.

LIU, P. V. 1962 . Non-motile varieties of Pseudomonas aeruginosa producing melanin-like pigment. J. Bact., 84, 378.

ManN, S. 1969. Über melaninbildende Stämme von Pseudomonas aeruginosa. Arch. Mikrobiol., 65, 359.

Meader, P. D., Robinson, G. H. And Leonard, V. 1925. Pyorubrin, a red water-soluble pigment characteristic of B. pyocyaneus. Amer. J. Hyg., 5, 682 .

Narano, Y. 1965 . Studies on red pigments production of Pseudomonas aeruginosa. Report I. J. Kansai med. Sch., 17, 240.

Narano, Y. 1966. Studies on red pigments production of Pseudomonas aeruginosa (strain no. 145). Report III. J. Kansai med. Sch., 18, 11.

Nicolaus, R. A., Piattelli, N. AND Fattorusso, E. 1964. The structure of melanins and melanogenesis. IV. On some natural melanins. Tetrahedron, 20, 1163.

PhILliPs, I. 1969. Identification of Pseudomonas aeruginosa in the clinical laboratory. J. med. Microbiol., 2, 9.

SchmidLI, B. 1955. Über Melanine, die dunklen Haut- und Haarpigmente. Helv. chim. Acta, 38, 1078.

SHORTER OXFORD ENGLISH DICTIONARY. 1965. 3rd ed., Oxford, p. 1230.

Simon, R. D. 1956. The use of fermentation reactions and pigment production to differentiate between types of Pseudomonas pyocyanea and other Pseudomonas species, especially fluorescens. Br. J. exp. Path., 37, 494.

WaHBA, A. H. 1965. Pyorubrin-producing Pseudomonas aeruginosa. Appl. Microbiol., 13, 291.

Witson, G. S. AND Mines, A. A. 1964. Topley and Wilson's Principles of bacteriology and immunity, 5th ed., London, p. 639.

YABUUCH, E. AND OHYAMA, A. 1972. Characterization of "pyomelanin "-producing strains of Pseudomonas aeruginosa. Int. J. syst. Bact., 22, 53. 